\title{
Coexister avec la résistance Éditorial aux antibiotiques : une réalité internationale en 2010 Laurent Gutmann, Olivier Lortholary
}

\section{Des mécanismes de résistance toujours plus sophistiqués...}

La résistance aux antibiotiques, qui existe de manière naturelle chez certaines bactéries vis-à-vis de certains antibiotiques, s'est considérablement accrue chez la plupart des espèces retrouvées en clinique depuis l'apparition des antibiotiques il y a plus de 70 ans. Certains pourraient même dire que c'est la preuve de l'activité de ces médicaments. Dans ce contexte, il est important de souligner que la plupart des antibiotiques sont des substances naturelles qui ont été modifiées pour améliorer leur spectre ou leur pharmacocinétique. Les microorganismes producteurs de ces antibiotiques ont élaboré des stratégies de défenses (mécanismes de résistance) pour ne pas être eux-mêmes victimes de leur propre production d'antibiotique. Ainsi retrouve-t-on déjà dans l'environnement les facteurs (enzymes modificatrices des antibiotiques par exemple) qui vont être par la suite sélectionnés et adaptés pour modifier ou détruire les antibiotiques utilisés en clinique. On aurait pu imaginer que des antibiotiques synthétiques ayant des cibles particulières, comme les quinolones, auraient mieux échappé à cette résistance une fois mis sur le marché. Néanmoins, comme pour beaucoup d'antibiotiques, c'est par le biais d'une mutation de la cible que sont apparus dès leur mise sur le marché des mutants résistants. Si la cible est unique et que sa modification n'a pas de retentissement important sur la physiologie de la bactérie, la sélection peut être facile (streptomycine vis-à-vis de Mycobacterium tuberculosis par exemple). À l'inverse, lorsqu'il existe plusieurs cibles, cette résistance peut être sélectionnée par paliers, les premiers entraînant des résistances de «bas niveau » difficiles à détecter (fluoroquinolones, oxazolidinones). De la même façon, la bactérie est capable de détourner ses propres processus physiologiques pour contrecarrer l'efficacité des antibiotiques: efflux actif (ex. Pseudomonas aeruginosa et fluoroquinolones), dérépression de gènes codant pour des enzymes (ex. Enterobacter spp. et céphalosporinase) ou encore accumulation de multiples mutations dans des système annexes. Ainsi, l'acquisition du phénotype de résistance de faible niveau aux glycopeptides chez les staphylocoques (GISA) ${ }^{1}$ associe un processus qui

${ }^{1}$ Glycopeptide intermediate-resistant Staphylococcus aureus. touche certes la paroi de la bactérie, mais aussi des systèmes de régulation dont les effets ne sont pas encore bien compris. Un moyen peu coûteux pour la bactérie est d'acquérir en bloc un système de résistance qu'elle peut soit intégrer dans son chromosome (S. aureus résistant à la méticilline), soit ajouter à un système préexistant (intégron), soit garder tel quel sur un plasmide, ceci aussi bien chez les bactéries à Gram négatif que chez les bactéries à Gram positif. Les antibiotiques synthétiques n'échappent pas à ce dernier mécanisme (résistance plasmidique de bas niveau par protection de la cible des fluoroquinolones).

\section{Diffusion accrue des bactéries et résistance : un risque immédiat}

Certaines bactéries se caractérisent par leur propension à diffuser dans la population humaine : c'est le cas du pneumocoque dont la sensibilité à la pénicilline est diminuée, chez les enfants, en l'absence d'une politique de maîtrise de l'utilisation des antibiotiques dans les infections respiratoires banales le plus souvent d'origine virale; c'est aussi le cas des clones de S. aureus résistants à la méticilline (SARM) communautaires, surtout aux États-Unis, mais aussi en Europe, et des SARM qui diffusent dans les hôpitaux en l'absence de mesures adéquates d'isolement. D'autres bactéries vont acquérir et diffuser ces mécanismes de résistance à partir de leur milieu naturel. Le tube digestif est probablement le plus grand échangeur de gènes de résistance aussi bien chez l'homme que chez l'animal. C'est là que sont réunies les conditions les plus appropriées à de tels échanges: la densité bactérienne y est importante, associant des espèces multiples cultivables ou non, résidentes ou de passage à partir du milieu extérieur, le tout associé à la pression des antibiotiques qui sont tous, à des degrés divers, éliminés par cette voie.

Les mécanismes de résistance ont évolué avec la sophistication des antibiotiques et leur apparition est directement en rapport avec la quantité globale d'antibiotiques utilisée dans la population communautaire et hospitalière, multipliant les risques que se rencontrent les événements nécessaires à leur apparition (mutation, acquisition, addition de ces mécanismes). Cette diversité des antibiotiques et les mécanismes génétiques des résistances qui leur sont associés sont à l'origine de la 
multirésistance que l'on retrouve dans la plupart des espèces, en particulier en milieu hospitalier mais aussi désormais de manière plus large et parfois en dehors de l'hôpital, dans les infections liées aux soins (SARM, entérocoques, Pseudomonas aeruginosa, Acinetobacter baumannii, et maintenant les entérobactéries incluant $\varepsilon$. coli). Un exemple caricatural est celui des bêta-lactamases à spectre étendu (BLSE) qui accompagnent souvent la résistance aux aminosides et aux fluoroquinolones. Le vrai problème est que ces enzymes touchent de nombreuses espèces bactériennes, qu'on les rencontre aussi bien en milieu hospitalier que communautaire et, qui plus est, que leur diffusion est mondiale ( $\varepsilon$. coli en particulier). L'importance accrue des échanges intercontinentaux a ajouté à la complexité du problème en permettant l'importation à bas bruit de résistances qui n'étaient pas communes jusqu'à présent. C'est le cas en particulier pour les bêta-lactamases à spectre étendu et les carbapénèmases. Dès lors, on pourrait envisager prochainement que la politique antibiotique empirique des états septiques sévères soit différente chez les personnes de retour de zones « endémiques pour ces mécanismes de résistance ».

\section{Quelles politiques de «bon usage des antibiotiques »?}

Dans ce contexte, deux situations paradoxales sont à mettre en exergue. Dans les pays développés, la mise en œuvre de politiques du bon usage des antibiotiques entraîne une réduction de l'usage des antibiotiques les plus récents, capables d'être actifs sur certaines espèces résistantes. Cette politique n'encourage pas les industriels à développer de nouveaux antibiotiques puisqu'ils ne sont pas assurés de leur rentabilité. À l'inverse, dans les pays où ces antibiotiques étaient trop coûteux, où la prescription médicale n'est pas une obligation pour leur délivrance, et qui n'ont pas les moyens de mettre en place une politique de bon usage, la capacité de développer des génériques pour des antibiotiques à très large spectre va devenir un facteur supplémentaire de sélection et accroître la résistance.

II serait inexact de dire que toutes les bactéries - en particulier celles qui sévissent à l'hôpital - sont résistantes ou multirésistantes, mais il est clair que dans les services spécialisés (réanimation) et chez certaines catégories de patients (mucoviscidose, patients multitraités), l'arsenal thérapeutique existant peut être défaillant. Le meilleur exemple est celui de l'escalade thérapeutique de première intention incluant les carbapénèmes et les glycopeptides lorsque survient une infection nosocomiale chez des patients de réanimation et la nécessité d'utiliser en dernier recours un antibiotique comme la colistine avec les incertitudes qui l'accompagnent. La situation est suffisamment sérieuse pour que l'on se donne tous les moyens de suivre ces résistances, localement puisqu'elles vont déterminer les antibiothérapies de première intention, mais aussi sur le plan national tant en ville qu'à l'hôpital. La recherche de nouveaux antibiotiques (actifs sur les entérocoques et staphylocoques résistants aux glycopeptides, sur Pseudomonas aeruginosa ou Mycobacterium tuberculosis totorésistants) ou de nouvelles stratégies anti-infectieuses associées (vaccin, molécules facilitant la pénétration des antibiotiques, etc.) est une nécessité. Elle pose le problème de l'intervention du politique pour imposer ou soutenir cette recherche avant que la situation elle-même ne l'impose (résistance en milieu communautaire en particulier) mais alors avec quel retard!

En attendant, la formation des soignants reste un atout majeur pour prévenir la diffusion et l'apparition des résistances dans les pays développés, mais aussi dans les pays à fort potentiel de développement. On pense ici à la mise en place de règles du bon usage et de stratégies d'utilisation des anti-infectieux, à la prévention des infections nosocomiales, à l'identification et au suivi des résistances pour les micro-organismes impliqués dans les pathologies les plus fréquentes. Le suivi des échanges interespèces doit être mis en place comme le bon usage des thérapeutiques anti-infectieuses chez l'animal. Cela souligne encore la nécessité de développer de nouveaux tests de détection rapide de la résistance et d'utilisation facile pour identifier ces résistances, et des centres de référence susceptibles de colliger leur évolution. Cet ensemble doit s'inscrire dans une politique locale, nationale et internationale. Depuis leur introduction au XXe siècle, les antibiotiques, l'une des plus belles découvertes médicales, sauvent des millions de vies humaines, mais coexister avec la résistance à ces molécules et essayer de la gérer au mieux est devenue une règle en 2010.

C'est l'objectif de ce numéro thématique de Médecine/Sciences que de sensibiliser le lecteur à quelques grands exemples préoccupants de résistance aux antibiotiques chez des bactéries que nous côtoyons au quotidien dans notre pratique clinique. $\diamond$

Antibiotic resistance: a world concern in 2010

\section{CONFLIT D'INTÉRÊTS}

Les auteurs déclarent n'avoir aucun confit d'intérêts concernant les données publiées dans cet article.

L. Gutmann: Laboratoire de microbiologie, hôpital européen Georges Pompidou, 20, rue Leblanc, 75908 Paris Cedex 15, France.

laurent.gutmann@egp.aphp.fr

0. Lortholary: Service des maladies infectieuses et tropicales, Centre d'infectiologie Necker-Pasteur,

Université Paris-Descartes, Hôpital Necker-Enfants Malades, 149, rue de Sèvres, 75743 Paris Cedex 15, France; Centre national de référence mycologie et antifongiques, unité de mycologie moléculaire, Institut Pasteur, Paris, France. olivier.lortholary@nck.aphp.fr

\section{TIRÉS À PART}

L. Gutmann et

0. Lortholary 